\section{ENHANCE YOUR KNOWLEDGE OF AESTHETIC ORTHODONTICS}

Become a member of the European Society of Aesthetic Orthodontics (ESA0) today and enhance your knowledge and experience of aesthetically focused orthodontics.

The ESAO offers an unbiased, specialised perspective on the provision of orthodontics, including events and conferences with speakers from across the profession.

Offering both Associate and Full membership, the benefits of joining the ESAO include:

- Access to the ESAO informed consent and orthodontic assessment forms

- A monthly newsletter

- Discounts on ESAO courses

- Discounts from the ESAO partners

- Access to online training materials

- Listing on the online dentist locator

- ESAO full membership logo.

On 21 June the ESAO will be running parallel courses for dentists and dental nurses with handson sessions. Dentists will get the opportunity to practise interproximal reduction (IPR), direct wire retainer bonding, composite edge bonding on typodonts, as well as assessing for TMJ abnormalities. Dental nurses will have hands-on experience in photography, impression taking and manufacturing vacuum formed retainers.

Contact the ESAO today or visit the website to find out more. Visit www.esao.co.uk or email esao@ esao.co.uk.

\title{
CONVENIENT ACCESS TO FREE DISINFECTION CPD
}

As busy dental professionals it can be difficult finding time to leave the practice and attend courses to gain necessary CPD hours. Now, infection control specialists Dentisan have added a CPD module to their website, enabling the whole dental team to easily access free, verifiable CPD whilst at the practice or even from the comfort of their own home.

The website now features articles that are published bi-monthly and are designed to assist dental professionals in meeting their disinfection and decontamination CPD requirements, one of the three core subjects that the GDC strongly recommends are completed.

After reading the article you are invited to answer a series of online multiple choice CPD questions, which once completed and submitted result in immediate feedback as to whether the answers given are correct. If necessary, you have the chance to re-answer. A certificate is issued in the form of a downloadable PDF, which can be used as evidence of CPD hours.

Each online CPD article is archived, enabling users to revisit should further clarification be required, allowing users to learn at a time and pace that suits their individual needs.

To see the latest CPD article visit www.dentisan. co.uk. If you would like to receive an email alert when new articles are added, please email your details tosample@dentisan.co.uk.

\section{GET THE MOST OUT OF YOUR INSTRUMENTS}

DENTSPLY's Cavitron range features a selection of ultrasonic scalers with sustained performance technology for easier and quicker removal of supra- and subgingival calculus that assures better clinical efficiency. To ensure the best results for your specific scaling needs, DENTSPLY has an extensive range of Cavitron inserts, each designed to achieve optimum performance across a variety of different procedures.

Innovatively designed to handle different levels of deposits, the Cavitron family of inserts offers a broad selection of tips that provide increased scaling and deplaquing performance. Each insert features the Focused Spray (FSI) water delivery system to flush away debris, enhancing visibility of the working area and improving patient comfort.

The standard size PowerLINE range efficiently removes heavier deposits and calculus bridges and extrinsic staining, whilst the SlimLINE series, which is $30 \%$ thinner than PowerLINE, improves subgingival access and adapts to the root anatomy, making it ideal for use in furcations and concavities. The THINsert delivers ultrasonic power with a tip that is $47 \%$ thinner than SlimLINE and offers a unique $9^{\circ}$ backbend that allows access to difficult areas such as misalignments and interproximal surfaces.

One insert is not enough to achieve success with ultrasonic scaling and the Cavitron family of inserts offers a broad selection of tips to provide optimal performance in specific indications.

To find out more about Cavitron inserts contact DENTSPLY UK on 08000723313 or visit www.

dentsply. co.uk.

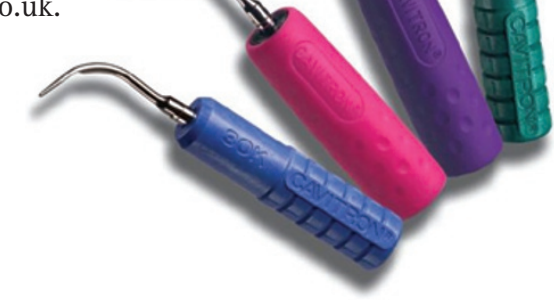

\section{IMPRESSIONS THAT 'SNAP-SET'}

Impregum Penta Soft Quick impression material is the latest in the line of 3M ESPE's pioneering polyether impression materials.

It features dimensional stability and the capacity to retain high levels of accurate detail. This reliable material delivers an accurate impression even under difficult clinical conditions, which results in the high precision fit of your restoration. Impregum Penta Soft Quick impression material is also easy and convenient to use, as it presents with a 'snap-set' behaviour, which sees the material set quickly as soon as the working time has passed.

Discover the newest product of 3M ESPE's biggest selling brand in impressioning in the UK - ask your 3M ESPE customer care representative about Impregum Penta Soft Quick impression material today.

For more information, call 0845 6025094 or visit www.3Mespe. co.uk.

3M ESPE, Impregum and Penta are trademarks of the $3 \mathrm{M}$ company. 